\title{
Total aortic arch replacement using elephant trunk or frozen elephant trunk technique: a case-control matching study
}

\author{
Sabreen Mkalaluh ${ }^{1}$, Marcin Szczechowicz ${ }^{1}$, Ahmed Mashhour ${ }^{1}$, Konstantin Zhigalov $^{1}$, Jerry Easo ${ }^{1}$, Harald \\ Christian Eichstaedt ${ }^{1}$, Jürgen Ennker ${ }^{1}$, Rohit Philip Thomas ${ }^{2}$, Ajay Chavan ${ }^{2}$, Alexander Weymann ${ }^{1}$ \\ ${ }^{1}$ Department of Cardiac Surgery, ${ }^{2}$ Department of Radiology, European Medical School Oldenburg-Groningen, Carl von Ossietzky University \\ Oldenburg, Oldenburg, Germany \\ Contributions: (I) Conception and design: S Mkalaluh, M Szczechowicz, A Mashhour, K Zhigalov, J Easo, A Weymann; (II) Administrative support: \\ All authors; (III) Provision of study materials: S Mkalaluh, M Szczechowicz, A Mashhour, K Zhigalov, J Easo, H Eichsteadt, A Weymann; (IV) \\ Collection and assembly of data: S Mkalaluh, M Szczechowicz, A Mashhour, K Zhigalov, J Easo, H Eichsteadt, J Ennker, A Weymann; (V) Data \\ analysis and interpretation: All authors; (VI) Manuscript writing: All authors; (VII) Final approval of manuscript: All authors. \\ Correspondence to: Sabreen Mkalaluh, MD. Department of Cardiac Surgery, European Medical School Oldenburg-Groningen, Carl von Ossietzky \\ University Oldenburg, Rahel-Straus-Straße 10, 26133 Oldenburg, Germany. Email: sabreen.mkalaluh@gmx.de.
}

\begin{abstract}
Background: Surgical management of aortic arch diseases is one of the most challenging issues in cardiovascular surgery. The aim of this study was to compare the outcome after frozen elephant trunk (FET) with conventional elephant trunk (ET) technique.

Methods: Out of a total of 551 patients after thoracic aortic surgery, we analyzed 70 consecutive patients, who underwent aortic arch replacement with ET or FET technique between 2001 and 2017 in our institution. The patients were case-control matched in regard to such variables as age, sex, presence of an acute aortic dissection and necessity for concomitant procedures. The analysis included 25 patient pairs.

Results: Among the 25 FET patients, eleven patients were female, the median age was 69, 15 (60\%) patients had an aortic dissection and thirteen needed various concomitant procedures. In the second group, treated with conventional ET technique, 10 (40\%) patients were female, the median age was 66 years, thirteen presented with an aortic dissection and 16 (64\%) underwent concomitant procedures. These and other characteristics did not differ significantly between the groups. In-hospital mortality was statistically similar: 5 (20\%) in the FET group vs. 8 (32\%) for ET group ( $\mathrm{P}=0.52)$. The incidence of stroke, acute renal failure and postoperative bleeding was comparable. The length of stay in the intensive care unit did not differ between the cohorts $(\mathrm{P}=0.258)$. Predictors of in-hospital mortality were length of the operation, bleeding postoperatively, and acute renal failure. The one-year survival rates were higher in the FET cohort compared to the conventional approach $(60 \%$ vs. $38 \%)$, however without statistical significance.

Conclusion: In regard to the short- and mid-term outcome, there were only slight differences between both techniques. In patients with extensive aneurysmal aortic disease, conventional ET and FET procedures seem to be associated with acceptable satisfactory mid-term outcome.
\end{abstract}

Keywords: Aortic surgery; elephant trunk (ET); outcome; case-control matching study

Submitted Jul 26, 2018. Accepted for publication Sep 30, 2018.

doi: $10.21037 /$ jtd.2018.10.42

View this article at: http://dx.doi.org/10.21037/jtd.2018.10.42

\section{Introduction}

The treatment of complex aortic pathologies, including the aortic arch and the thoracic descending aorta remains a surgical challenge. Up to the early 2000s, multistage repair was often necessary for completion of the treatment (1). Borst et al. introduced the elephant trunk (ET) technique in 1983 and its aim was to facilitate the second-stage intervention of the descending aorta via lateral thoracic 
or thoracoabdominal approaches (2). The most important change was the introduction of a stent attached distally to the Dacron graft, which seals the aneurysm of the descending aorta. This modified technique was named "frozen elephant trunk" (FET) and found its way rapidly into clinical use (3).

After repair of the aortic arch and proximal descending aorta with the FET technique, the second-stage can be performed through a thoracic endovascular aortic repair (TEVAR) or open aortic surgery with similar complications and mortality rates (4). Various prostheses have been developed in Europa for FET technique. The E-Vita Open Plus (Jotec GmbH, Hechingen, Germany) was the first commercially available hybrid prosthesis (1). The most widely used Thoraflex hybrid device (Vasutek Terumo, Inchinnan, Scotland, UK) was introduced in 2012 (1). Several studies reported comparable in-hospital mortality and postoperative complications after both techniques (4-7). The aim of this study was to compare the outcomes and short- and mid-term survival in patients who had undergone the conventional ET or the FET procedures.

\section{Methods}

\section{Study population}

During the period from 2001 to 2017, a total of 266 total aortic arch and descending aorta procedures were performed, of which 45 as conventional ET and 25 as FET procedures.

Postoperative outcomes and major complications such as in-hospital mortality, stroke, exploration for bleeding, re-intubation, blood product use, acute renal failure, atrial fibrillation were analyzed.

\section{Definitions}

We divided the complications into primary and secondary complications. The primary complication was in-hospital mortality. Secondary complications included for example, neurological deficits and respiratory failure.

In-hospital mortality was defined as death within 30 days after surgery or death during the original hospitalization. Postoperative temporary dialysis was defined as a new necessity for dialysis during the hospital stay. The dialysis was described as permanent if it was still necessary at the time of discharge. Postoperative stroke, delirium, paraparesis, and paraplegia were defined as a new and permanent or temporary neurological disability or deficit. Postoperative respiratory failure was defined as reintubation or tracheotomy. Gastrointestinal complications included gastrointestinal bleeding and visceral ischemia. Redo-operation was defined as surgery on a previously operated aorta.

Follow-up data were obtained during clinical visits and via telephone correspondence.

\section{Statistical analysis}

For comparison, the case-control matched groups were created basing on the following variables: sex, age, presence of acute aortic dissection and need for concomitant procedures. The patients were divided into two groups: those who received conventional ET or FET; 25 pairs were generated. The statistical analyses were done using IBM SPSS, version 24 (IBM Corp.). Categorical data are described as percentages. Continuous variables are presented as mean \pm standard deviation for normal distribution or the median with quartiles for non-normal distribution. Statistical significance was defined as $\mathrm{P}<0.05$. The mean values were compared using Student $t$-test for normally distributed variables and the Mann-Whitney U-test for variables that were not normally distributed. Predictors of in-hospital mortality were analyzed using logistic regression and the results are presented as odds ratios (ORs) with $95 \%$ confidence intervals (CIs). We searched for predictors of mid-term mortality using proportional hazard regression and the results are showed as hazard ratios (HRs) with $95 \%$ CI. Cumulative survival was described using the KaplanMeier-method. Breslow and log-rank tests were used for survival comparison.

\section{Surgical technique}

Our surgical approaches have been previously described in detail $(1,6,8)$. All patients were operated on through median sternotomy. All operations were performed using cardiopulmonary bypass (CPB). We used in all cases cold crystalloid cardioplegic solution and selective antegrade cerebral perfusion. The first stage of the conventional ET technique is similar to standard surgery of the ascending aorta and aortic arch. The second stage is like standard descending thoracic or thoracoabdominal aortic aneurysm repair. In the case of the FET technique, after median sternotomy, antegrade selective cerebral perfusion and systemic heparinization, a guide-wire is inserted through 
Table 1 Preoperative patients' characteristics

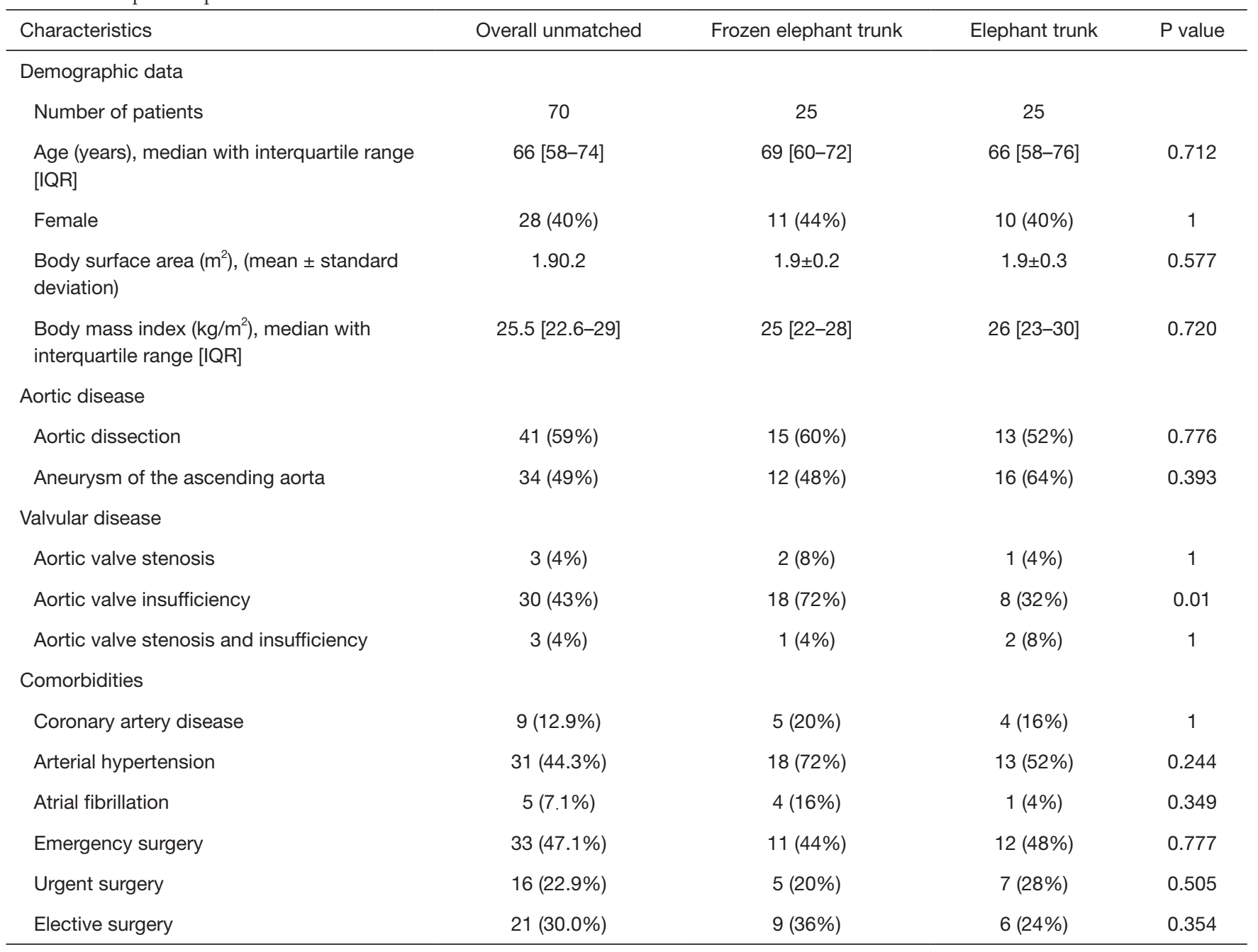

the femoral artery in the true lumen of the descending aorta. The arterial cannulation sites were differently, the right axillary artery or the innominate artery. The venous drainage was achieved mostly by cannulation of femoral vein in cases of redo surgery or the right atrium. The desired temperature in the convention ET cohort was $20^{\circ} \mathrm{C}$. In the FET cohort, the circulatory arrest was performed at a median temperature of $25^{\circ} \mathrm{C}$.

\section{Results}

\section{Baseline characteristic}

The median age of the patients was 69 years in the FET group versus 66 years in the conventional ET group $(\mathrm{P}=0.712)$. The male gender was predominant in both groups. In $8 \%$ of patients in each group, the procedure was a redo operation. Sixty percent of the FET cohort versus $52 \%$ of the ET cohort were operated because of aortic dissection (acute and chronic), ( $\mathrm{P}=0.776)$. The etiology of the aortic disease was atherosclerotic aneurysm. More demographic characteristics of the case-control matched cohorts are outlined in detail in Table 1.

\section{Operative characteristics}

In both cohorts, the median operation times as well as the CPB times were similar. Additionally, the crossclamp times were not significantly longer in the FET than in the conventional ET group $(\mathrm{P}=0.16)$. The time of deep hypothermic circulatory arrest differ significantly between the cohorts, $42 \pm 33$ minutes for the FET versus 
Table 2 Intraoperative data

\begin{tabular}{|c|c|c|c|c|}
\hline Characteristics & Overall unmatched & Frozen elephant trunk & Elephant trunk & $P$ value \\
\hline $\begin{array}{l}\text { Operation (min), median with } \\
\text { interquartile range [IQR] }\end{array}$ & 335 [293-409] & 335 [295-409] & 305 [270-401] & 0.459 \\
\hline $\begin{array}{l}\text { Cardiopulmonary bypass (min), } \\
\text { mean } \pm \text { standard deviation }\end{array}$ & $239 \pm 59$ & $229 \pm 53$ & $229 \pm 60$ & 0.260 \\
\hline $\begin{array}{l}\text { Hypothermia }\left({ }^{\circ} \mathrm{C}\right) \text {, median with } \\
\text { interquartile range }[\mathrm{IQR}]\end{array}$ & 24 [20-25] & 25 [23-26] & 20 [20-24] & 0.109 \\
\hline $\begin{array}{l}\text { Circulatory arrest (mean } \pm \text { standard } \\
\text { deviation) }\end{array}$ & $33.5 \pm 31$ & $42 \pm 33$ & $36 \pm 29$ & $<0.001$ \\
\hline Aortic valve replacement & $3(4 \%)$ & $2(8 \%)$ & $1(4 \%)$ & 1 \\
\hline CABG & $12(17 \%)$ & $5(20 \%)$ & $2(8 \%)$ & 0.417 \\
\hline
\end{tabular}

CABG, coronary artery bypass grafting.

$36 \pm 29$ minutes in the conventional cohort, $\mathrm{P}<0.001$. The surgical data were presented in Table 2.

\section{Clinical results}

The in-hospital outcomes and complications are summarized in Table 3. In terms of in-hospital mortality, there was no significant difference between the investigated groups (conventional ET: $n=8 ; 32 \%$ versus $n=5 ; 20 \%$ in FET; $\mathrm{P}=0.520$ ). The majority of early death cases suffered from acute aortic dissection. In the FET group, stroke occurred in $24 \%(n=6)$ versus $8 \%(n=2)$ of patients in the conventional ET group $(\mathrm{P}=0.123)$. Symptomatic transitory psychotic syndrome was observed in 5 patients $(20 \%)$ in the FET group, whereas its incidence in the conventional ET group was not significantly lower $(n=3 ; 12 \% ; P=0.702)$. Moreover, there were 3 cases $(12 \%)$ of pericardial tamponade in each group $(\mathrm{P}=1)$. Exploration rate due to mediastinal bleeding did not differ significantly between the matched groups $(\mathrm{P}=1)$. The transfusion requirements were significantly lower in the FET patients $(\mathrm{P}=0.001)$. The incidence of postoperative respiratory failure requiring re-intubation $(\mathrm{P}=0.417)$ or tracheotomy $(\mathrm{P}=1)$ were comparable. The median time of ventilation of the FET cohort was 143 hours versus 71 hours in the conventional ET patients, although the difference was not statistically relevant $(\mathrm{P}=0.507)$. The rate of acute renal failure requiring dialysis was very similar in both groups. The median intensive care length of stay (ICU-LOS) was 8.5 days after FET and 8 days after conventional ET. This difference was not statistically significant, as well $(\mathrm{P}=0.258)$.

The logistic regression analysis for both techniques could not identify, age, gender, acute aortic dissection, CPB and cross-clamp times, concomitant procedures, re-intubation, stroke, and ICU-LOS as predictors of in-hospital mortality. Only acute renal failure requiring dialysis, mediastinal bleeding and length of the procedure were identified as predictors of in-hospital mortality. In the proportional hazard regression, only acute renal failure requiring dialysis seems to be a predictor of overall mortality $(\mathrm{P}=0.004)$. The procedures per se did not influence neither the in-hospital nor the overall mortality. More details on predictors of in-hospital and overall mortality are presented in Table 4.

\section{Follow-up data}

The survival rates are illustrated on Figure 1. The mean follow-up time was 791 days. One-year survival rates were $38 \%$ after conventional procedure versus $60 \%$ after the FET procedure, respectively ( $\log$ rank $\mathrm{P}=0.118$, Breslow $\mathrm{P}=0.039$ ). 
Table 3 Early outcome and postoperative complications

\begin{tabular}{|c|c|c|c|c|}
\hline Characteristics & Overall unmatched & Frozen elephant trunk & Elephant trunk & $P$ value \\
\hline $\begin{array}{l}\text { Transfusion of packed red cells (units), } \\
\text { median with interquartile range [IQR] }\end{array}$ & $6[2-15]$ & $8[2-16]$ & 6 & 0.001 \\
\hline Extracorporeal life support & $3(4 \%)$ & $1(4 \%)$ & $1(4 \%)$ & 1 \\
\hline \multicolumn{5}{|l|}{ Postoperative adverse events } \\
\hline Stroke & $17(24 \%)$ & $6(24 \%)$ & $2(8 \%)$ & 0.123 \\
\hline Delirium & $9(13 \%)$ & $5(20 \%)$ & $3(12 \%)$ & 0.702 \\
\hline Revision for bleeding & $12(17 \%)$ & $4(16 \%)$ & $5(20 \%)$ & 1 \\
\hline Pericardial tamponade & $7(10 \%)$ & $3(12 \%)$ & $3(12 \%)$ & 1 \\
\hline Acute renal failure requiring dialysis & $15(21 \%)$ & $6(24 \%)$ & $6(24 \%)$ & 1 \\
\hline Re-intubation & $9(13 \%)$ & $5(20 \%)$ & $2(8 \%)$ & 0.417 \\
\hline Tracheotomy & $13(19 \%)$ & $6(24 \%)$ & $5(20 \%)$ & 1 \\
\hline Atrial fibrillation & $18(26 \%)$ & $7(28 \%)$ & $6(24 \%)$ & 1 \\
\hline Cardiopulmonary resuscitation & $4(6 \%)$ & $1(4 \%)$ & $3(12 \%)$ & 0.609 \\
\hline In-hospital mortality & $19(27 \%)$ & $5(20 \%)$ & $8(32 \%)$ & 0.520 \\
\hline
\end{tabular}

\section{Discussion}

The development of the ET procedure (conventional and frozen) is claimed to be the greatest improvement in the treatment of complex aortic surgery (9). The FET technique offers the option of definitive treatment of combined and extensive lesions in a single-stage procedure (3). The FET technique indications include several conditions such as Type A and B, acute and chronic aortic dissections as well as atherosclerotic aneurysms involving the aortic arch and the descending thoracic aorta $(1,10)$. Due to the encouraging short- and mid-term results of the FET, this technique has become increasingly common (11-13). The overall inhospital mortality rates after FET procedures were reported between $1.8 \%$ and $17.2 \%(4,5,10,11,14-16)$. On the other hand, the 30-day mortality after conventional ET, stage I, ranged between $5.1 \%$ to $12 \%$, and for stage II between $4 \%$ and $9.7 \%(5-7,17)$. During the time awaiting the stage
II of conventional ET, mortality rates between $2 \%$ and $11.7 \%$ were observed, with frequent death on rupture of the descending thoracic aorta $(6,7,18,19)$.

Di Eusanio et al. reported a $4.8 \%$ in-hospital mortality in the cohort of FET versus $13.9 \%$ in the conventional technique in a comparative study. However, these differences were not statistically significant (20). In our cohort, we reported remarkably higher mortality rates than above mentioned, $20 \%$ in conventional cohort versus $32 \%$ in FET cohort, without statistically significant difference. The reason of these high mortality rates could be explained with the high rates (>50\%) of emergency and urgent surgery in our cohort. Schepens et al. reported $7.3 \%$ inhospital mortality in elective setting versus $25 \%$ in-hospital mortality in emergent cases after conventional ET (17).

The most feared complications after aortic surgery are stroke and spinal cord injury. In our case-control 
Table 4 Predictors of mortality

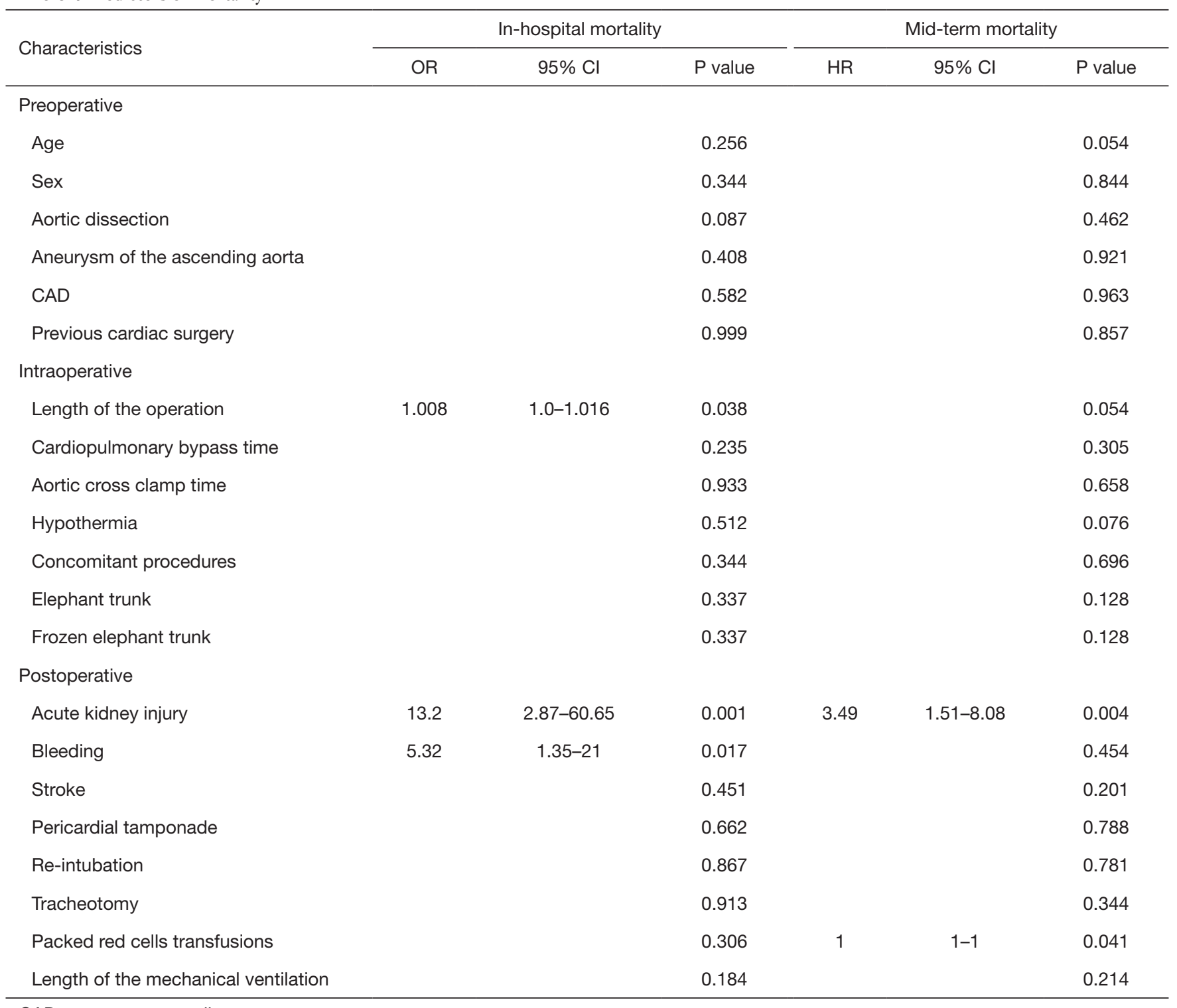

$\mathrm{CAD}$, coronary artery disease.

matched analysis, we observed higher stroke rates in the cohort of FET, however, without statistically significant differences between the techniques. Compared with our results, Leontyev et al. showed higher stroke rates after conventional ET (16\% versus 13\%), likewise without significantly difference (15). In contrast to these facts regarding the incidence of strokes, Leontyev et al. could show in the same study, that the occurrence of new-onset paraplegia was significantly higher in the FET group (21.7\% vs. $4.0 \%, \mathrm{P}<0.001)$, and aortic repair with the FET technique was an independent predictor for paraplegia (15).
These findings could not be supported by our case series.

In several studies, the incidence of paraplegia was often lower after the conventional ET technique than after the FET technique. Incidences between $1 \%$ to $21.7 \%$ for the FET were reported versus $2.9 \%$ to $4.8 \%$ for the conventional ET technique $(5,14,15,20)$.

In regard to other postoperative complications such as reopening due to mediastinal bleeding, prolonged ventilation support or acute renal failure requiring dialysis, we presented similar outcomes after both techniques, which is comparable with results reported in the recent 

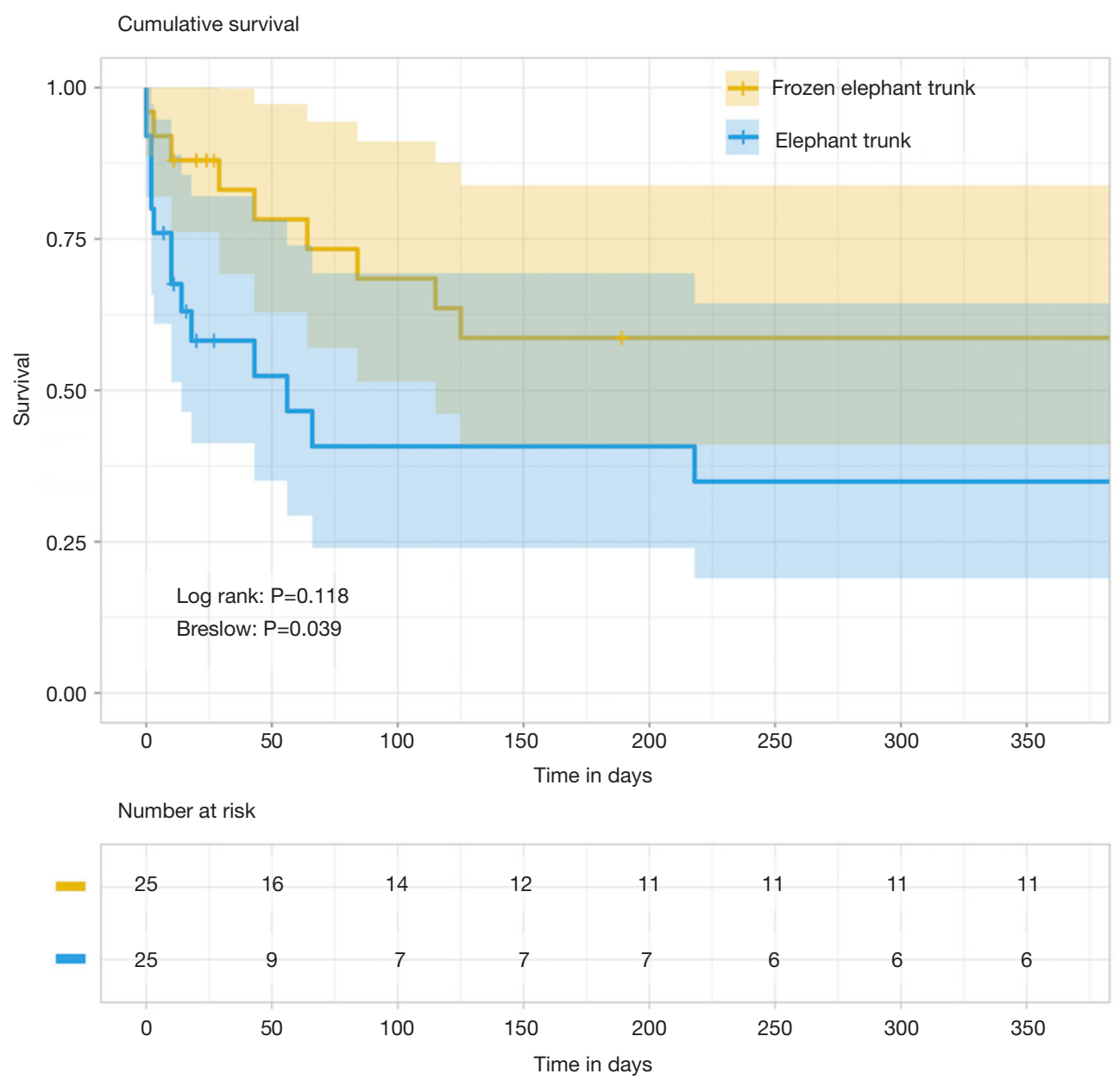

Figure 1 Cumulative survival of both patient cohorts. Kaplan-Meier curve.

literature (11,14-16). According to a multicenter study with 240 patients from the E-Vita registry, there exists a significant association between in-hospital mortality and a serum creatinine concertation above $2 \mathrm{mg} / \mathrm{dL}$, a need for permanent or temporary dialysis and prolonged intubation time $>72$ hours (11). Martens et al. confirmed in their analysis that postoperative dialysis and reopening for bleeding are also risk factors of mortality after total aortic arch repair (90\% of included patients received conventional or FET) (21). Furthermore, Leontyev et al. showed, that type A aortic dissection was an independent predictor of inhospital mortality (15). In our case-control matched cohorts, acute renal failure requiring permanent or temporary dialysis and bleeding complications were associated with significantly higher in-hospital mortality rates. Among our patients, type A aortic dissection was overrepresented and a high rate of patients with aortic dissection died in hospital, although, it could not be identified as predictor of in-hospital mortality.

In seven patients in our collective, coronary artery bypass grafting (CABG) was performed as a concomitant procedure. There was no significant influence on the outcome after both surgical techniques. LeMaire et al. reported that concomitant coronary artery bypass grafting increased the early mortality as well as the incidence of stroke after conventional ET (5). Another study showed that concomitant CABG was a risk factor of overall mortality in surgical repair of aortic arch, including conventional and frozen ET procedures (22). In our small sample, we could not confirm this statement. In our collective, we could not identify hypothermia as predictor of in-hospital mortality as well as mid-term mortality in ET techniques. Janczyk et al. reported that hypothermia is a strong independent contributor to mortality in patients with ruptured abdominal aortic aneurysms (23). In the treatment of acute 
and chronic thoracic aortic pathology, hypothermia did not directly affect mortality; rather, the duration of hypothermia was the relevant factor (24). Czerny et al. investigated the influence of age on mortality and incidence of neurological injury in patients undergoing surgical repair with hypothermic circulatory arrest in acute and chronic thoracic aortic pathology in a collective of 523 patients. This study revealed that hypothermic circulatory arrest $\geq 40$ minutes was an independent predictor of mortality (25). In another study with 490 patients, Ziganshin et al. showed in their multivariate analysis that deep hypothermic circulatory arrest time $>50$ minutes and aortic dissection were strong predictors of composite adverse outcomes. The stroke rate was $1.3 \%$ for patients with a deep hypothermic circulatory arrest time $<50$ minutes, significantly different from the $16.7 \%$ stroke rate for whose with a deep hypothermic circulatory arrest time longer than 50 minutes (24).

A great number of patients need a second-stage procedure for repair completion. After FET, more lessinvasive endovascular interventions are possible, because of more secure landing zone, which is offered through the FET prostheses (4). This fact could explain the lower midterm mortality rates after the FET procedures. In regard to conventional technique, there is a relevant mortality risk within the interval between the therapy stages, which is almost between $2 \%$ and $11.7 \%(6,7)$. Additionally, after completed second stage repair, the reported operative mortality within 30 -day was between $4 \%$ and $9.7 \%(5,6,26)$. The waiting time and the second stage of the repair increase the cumulative mortality risk. So, the main advantage of FET remains the possibility of single-stage treatment in many cases and risk reduction of additional distal aortic surgery. This aspect makes remarkable differences in early mortality in several studies, however, the mid-term survival did not differ significantly (4).

Another interesting aspect is that in one meta-analysis, the FET technique was followed by partial or complete thrombosis of a persistent false lumen in $88.9 \%$ cases of acute type A aortic dissection. The FET technique promotes restoring the flow in the true lumen and covering proximal entry tears because of antegrade stenting of the distal thoracic aorta, and these aspects can possibly slow down the aortic remodeling (27).

\section{Limitation}

Our study had various limitations. It was a single-center retrospective study with a small sample. Furthermore, we did not have long-term data about survival and the need of re-operations and endovascular interventions.

\section{Conclusions}

In patients with complicated aortic diseases, both conventional ET and FET seem to be comparable techniques with satisfactory early outcomes. We were able to present a rare case-control matching analysis that compares the conventional ET and FET procedures.

\section{Acknowledgements}

None.

\section{Footnote}

Conflicts of Interest: The authors have no conflicts of interest to declare.

Ethical Statement: The European Medical School Oldenburg-Groningen, Carl von Ossietzky University Oldenburg had approved this study on 22 May 2017. Number of IRB approval: 2017-040.

\section{References}

1. Di Marco L, Pantaleo A, Leone A, et al. The Frozen Elephant Trunk Technique: European Association for Cardio-Thoracic Surgery Position and Bologna Experience. Korean J Thorac Cardiovasc Surg 2017;50:1-7.

2. Borst HG, Walterbusch G, Shaps D. Extensive aortic replacement using "elephant trunk" prosthesis. Thorac Cardiovasc Surg 1983;31:37-40.

3. Karck M, Chavan A, Hagl C, et al. The frozen elephant trunk technique: a new treatment for thoracic aortic aneurysms. J Thorac Cardiovasc Surg 2003;125:1550-3.

4. Rustum S, Beckmann E, Wilhelmi M, et al. Is the frozen elephant trunk procedure superior to the conventional elephant trunk procedure for completion of the second stage? Eur J Cardiothorac Surg 2017;52:725-32.

5. LeMaire SA, Carter SA, Coselli JS. The elephant trunk technique for staged repair of complex aneurysms of the entire thoracic aorta. Ann Thorac Surg 2006;81:1561-9; discussion 1569.

6. Safi HJ, Miller CC 3rd, Estrera AL, et al. Staged repair of extensive aortic aneurysms: morbidity and 
mortality in the elephant trunk technique. Circulation 2001;104:2938-42.

7. Safi HJ, Miller CC 3rd, Estrera AL, et al. Staged repair of extensive aortic aneurysms: long-term experience with the elephant trunk technique. Ann Surg 2004;240:677-84; discussion 684-5.

8. Uchida N, Shibamura H, Katayama A, et al. Long-term results of the frozen elephant trunk technique for the extensive arteriosclerotic aneurysm. J Thorac Cardiovasc Surg 2010;139:913-7.

9. Di Bartolomeo R, Murana G, Di Marco L, et al. Frozen versus conventional elephant trunk technique: application in clinical practice. Eur J Cardiothorac Surg 2017;51:i20-28.

10. Shimamura K, Kuratani T, Matsumiya G, et al. Longterm results of the open stent-grafting technique for extended aortic arch disease. J Thorac Cardiovasc Surg 2008;135:1261-9.

11. Pacini D, Tsagakis K, Jakob H, et al. The frozen elephant trunk for the treatment of chronic dissection of the thoracic aorta: a multicenter experience. Ann Thorac Surg 2011;92:1663-70; discussion 1670.

12. Di Bartolomeo R, Pacini D, Savini C, et al. Complex thoracic aortic disease: single-stage procedure with the frozen elephant trunk technique. J Thorac Cardiovasc Surg 2010;140:S81-5; discussion S86-91.

13. Di Eusanio M, Armaro A, Di Marco L, et al. Short- and midterm results after hybrid treatment of chronic aortic dissection with the frozen elephant trunk technique. Eur J Cardiothorac Surg 2011;40:875-80.

14. Ius F, Fleissner F, Pichlmaier M, et al. Total aortic arch replacement with the frozen elephant trunk technique: 10-year follow-up single-center experience. Eur J Cardiothorac Surg 2013;44:949-57.

15. Leontyev S, Borger MA, Etz CD, et al. Experience with the conventional and frozen elephant trunk techniques: a single-centre study. Eur J Cardiothorc Surg 2013;44:107682; discussion 1083.

16. Shrestha M, Bachet J, Bavaria J, et al. Current status and recommendations for use of the frozen elephant trunk technique: a position paper by the Vascular Domain of EACTS. Eur J Cardiothorac Surg 2015;47:759-69.

17. Schepens MA, Dossche KM, Morshuis WJ, et al. The elephant trunk technique: operative results in 100 consecutive patients. Eur J Cardiothorac Surg 2002;21:276-81.
18. Castrovinci S, Murana G, de Maat GE, et al. The classic elephant trunk technique for staged thoracic and thoracoabdominal aortic repair: long-term results. J Thorac Cardiovasc Surg 2015;149:416-22.

19. Estrera AL, Miller CC 3rd, Porat EE, et al. Staged repair of extensive aortic aneurysms. Ann Thorac Surg 2002;74:S1803-5; discussion S1825-32.

20. Di Eusanio M, Borger M, Petridis FD, et al. Conventional versus frozen elephant trunk surgery for extensive disease of the thoracic aorta. J Cardiovasc Med (Hagerstown) 2014;15:803-9.

21. Martens A, Beckmann E, Kaufeld T, et al. Total aortic arch repair: risk factor analysis and follow-up in 199 patients. Eur J Cardiothorac Surg 2016;50:940-8.

22. Bashir M, Field M, Shaw M, et al. Influence on Early and Medium-Term Survival Following Surgical Repair of the Aortic Arch. Aorta (Stamford) 2014;2:56-73.

23. Janczyk RJ, Howells GA, Bair HA, et al. Hypothermia is an independent predictor of mortality in ruptured abdominal aortic aneurysms. Vasc Endovascular Surg 2004;38:37-42.

24. Ziganshin BA, Rajbanshi BG, Tranquilli M, et al. Straight deep hypothermic circulatory arrest for cerebral protection during aortic arch surgery: Safe and effective. J Thorac Cardiovasc Surg 2014;148:888-98; discussion 898-900.

25. Czerny M, Krahenbuhl E, Reineke D, et al. Mortality and neurologic injury after surgical repair with hypothermic circulatory arrest in acute and chronic proximal thoracic aortic pathology: effect of age on outcome. Circulation 2011;124:1407-13.

26. Svensson LG, Kim KH, Blackstone EH, et al. Elephant trunk procedure: newer indications and uses. Ann Thorac Surg 2004;78:109-16; discussion 109-16.

27. Di Eusanio M, Castrovinci S, Tian DH, et al. Antegrade stenting of the descending thoracic aorta during DeBakey type 1 acute aortic dissection repair. Eur J Cardiothorac Surg 2014;45:967-75.

Cite this article as: Mkalaluh S, Szczechowicz M, Mashhour A, Zhigalov K, Easo J, Eichstaedt HC, Ennker J, Thomas RP, Chavan A, Weymann A. Total aortic arch replacement using elephant trunk or frozen elephant trunk technique: a casecontrol matching study. J Thorac Dis 2018;10(11):6192-6200. doi: $10.21037 /$ jtd.2018.10.42 